\title{
MR889, a neutrophil elastase inhibitor, in patients with chronic obstructive pulmonary disease: a double-blind, randomized, placebo-controlled clinical trial
}

\author{
M. Luisetti*, C. Sturani**, D. Sella**, E. Madonini+, V. Galavotti **, G. Bruno+t, \\ V. Peona*, U. Kucich ${ }^{*}$, G. Dagnino++, J. Rosenbloom*, B. Starcher*, C. Grassi*
}

\begin{abstract}
MR889, a neutrophil elastase inhibitor, in patients with chronic obstructive pulmonary disease: a double-blind, randomized, placebo-controlled clinical trial. M. Luisetti, C. Sturani, D. Sella, E. Madonini, V. Galavotti, G. Bruno, V. Peona, U. Kucich, G. Dagnino, J. Rosenbloom, B.Starcher, C. Grassi. (CERS Journals Ltd 1996.

ABSTRACT: We investigated whether MR889, a synthetic cyclic thiolic elastase inhibitor, administered for a period of 4 weeks to chronic obstructive pulmonary disease (COPD) patients, is well-tolerated, and whether it modifies biochemical indices of lung destruction.

The study was a double-blind, randomized, placebo-controlled clinical trial in COPD patients. Thirty subjects were administered MR889 orally at a dose of 500 $\mathrm{mg}$ b.i.d. for 4 weeks, and 30 received placebo following the same schedule. In addition to safety parameters, MR889 efficacy was checked by a pretreatment/posttreatment evaluation of levels of plasma elastin-derived peptides and urinary desmosine.

There were no statistically significant differences between pretreatment and posttreatment efficacy parameter levels either in the control group or in the treated group. However, in a subset of treated patients with a short disease duration, the level of urinary desmosine dropped significantly with respect to pretreatment values $(\mathbf{p}=\mathbf{0 . 0 0 4})$.

We conclude that MR889 is safe to administer to COPD patients for a period of at least 4 weeks. During this time, MR889 does not modify biochemical markers of lung destruction in unselected COPD patients. Nevertheless, a subset of treated patients with fairly short disease duration showed a post-treatment reduction of desmosine urine levels, thus justifying the need for further studies to prove the efficacy of MR889 in modulating indices of lung destruction in COPD.

Eur Respir J., 1996, 9, 1482-1486.
\end{abstract}

*Istituto di Tisiologia e Malattie Respiratorie, Università di Pavia, IRCCS Policlinico San Matteo, Pavia, Italy. **Divisione di Pneumologia, Istituti Ospedalieri C. Poma, Mantova, Italy. +Ist. Villa Marelli, Milano, Italy. ${ }^{++}$Medea Research, Milano, Italy. ثUniversity of Pennsylvania School of Dentistry, Philadelphia, PA, USA. +\$Department of Biochemistry, University of Texas Health Center at Tyler, USA.

Correspondence: M. Luisetti, Istituto di Tisiologia e Malattie Respiratorie, IRCCS Policlinico San Matteo, Università degli Studi di Pavia, Via Taramelli 5, 27100 Pavia, Italy

Keywords: Chronic bronchitis, elastin, plasma elastin-derived peptides, pulmonary emphysema, urinary desmosine

Received: October 171995

Accepted after revision March 151996

This study has been supported by a research grant from Medea Research Ltd, a RC grant of IRCCS Policlinico San Matteo and a Ricerca Finalizzata grant from Regione Lombardia.
The imbalance between $\alpha_{1}$-proteinase inhibitor $\left(\alpha_{1}\right.$ PI), the major naturally occurring plasma serine proteinase inhibitor [1], and neutrophil elastase (NE), the potent proteinase discharged by triggered neutrophils, is the basis of the so-called proteinase/ inhibitor hypothesis of the pathogenesis of chronic obstructive pulmonary disease (COPD) [2]. Pulmonary emphysema in $\alpha_{1}$-PI sufficient COPD patients is believed to develop from the unrestrained digestion of lung interstitial elastin by NE, due to an impairment of $\alpha_{1}$-PI by either oxidants or proteinases [3-5].

The proteinase/inhibitor hypothesis represents, in turn, the basis of COPD therapeutic strategies specifically directed at the underlying cause of the disease $[6,7]$.

Administration of synthetic NE inhibitors is one of the possible strategies to be pursued in treating $\alpha_{1}$-PI sufficient COPD patients. Tight-binding, reversible inhibitors are believed to have greater therapeutic potential, compared to more efficient, but also more toxic inhibitors [8], since they are less likely to react in vivo with other proteins or nucleophils.
The cyclic thiolic compound MR889 (Midesteine; 3[3-thiophencarboxythio]-N-[ dihydro-2(3H)-thiophenone3-il]-propionamide), a derivative both of homocysteine lactone and thiolactic acid, is a reversible, slow-binding, fully competitive serine proteinase inhibitor, with a specific activity towards $\mathrm{NE}\left(K_{\mathrm{i}}=1.38 \mu \mathrm{M}\right)$ [9-11]. The MR889 three-dimensional structures studied by X-ray diffraction and nuclear magnetic resonance (NMR) techniques in solution and in the solid state show conformations displaying a fully accessible electrophil C(1) atom [12]. At the onset of the acylation reaction, the cyclic thiolic compound $\mathrm{C}(1)$ atom is properly reached by the target enzyme SER195 OG atom and the acylenzyme adducts contain the thiophenecarbonyl moiety of the inhibitor covalently bound to the SER195 OG atom [13, 14].

The elucidation of the inhibition mechanism of NE by cyclic thiolic compounds, coupled to the absence of significant toxic effects [9], prompted us to perform a clinical trial in COPD patients with two goals in mind, both crucial in designing a study on a NE inhibitory agent 
[15]. Firstly, to test the safety and the tolerability of this inhibitor in COPD patients. Secondly, to evaluate the possible in vivo proteinase inhibitory activity of MR889 by measuring changes in blood and urine concentration of elastin-derived peptides (EDP) and desmosine (DES), respectively, both indices of elastolysis and possibly related to lung destruction in vivo.

\section{Material and methods}

\section{Study design}

The study was designed as a randomized, double-blind, placebo-controlled clinical trial with a pretreatment/posttreatment analysis of a group of patients. For the primary response variables assumed, i.e. plasma elastin-derived peptides and urine desmosine, it was estimated that 30 patients in each group was an adequate sample size. In fact, this number would allow detection of changes in plasma elastin-derived peptides no smaller than $28 \%$ for probabilities of type I and II errors of 0.05 and 0.20 , respectively. For urine desmosine, 30 patients would be adequate to detect differences as small as $32 \%$ at power $=0.8$.

The study protocol was approved by the Ethics Committees of all Institutions involved.

\section{Characteristics of patients}

The clinical trial was performed with out-patients at the Divisione di Pneumologia, Istituti Ospedalieri C. Poma of Mantova (Italy). Sixty COPD patients, diagnosed according to American Thoracic Society (ATS) standards [16], were recruited if they fulfilled the following criteria: 1) irreversible airflow obstruction, defined as forced expiratory volume in one second (FEV1) less than $70 \%$ predicted and less than $20 \%$ reversible; 2) former cigarette smokers who had ceased for at least $1 \mathrm{yr}$; 3) aged 40-75 yrs; 4) absence of COPD exacerbations within the last 2 months, i.e. stable disease; 5) absence of other significant diseases, such as diabetes and severe heart, renal or hepatic failure; and 6) no use of either systemic or inhaled corticosteroids, antibiotics and nonsteroidal anti-inflammatory agents within the last 2 months. In addition, COPD patients with inherited $\alpha_{1}$-PI deficiency were excluded from the study.

The duration of the disease, based on the onset of respiratory symptoms, was carefully recorded, together with smoking history.

\section{Timing of events and treatments}

At the time of enrolment, patients underwent a complete physical examination (including measurement of heart and respiratory rates and blood pressure), pulmonary function testing, blood gas analysis, chest radiograph, electrocardiogram, complete blood and urine laboratory tests (including red and white cell and platelet counts, haemoglobin, haematocrit, aspartate aminotransferase (AST), alanine aminotransferase (ALT), blood urea, $\gamma$ glutamyl transferase $(\gamma-\mathrm{GT})$, bilirubin, creatinine, alkaline phosphatase, glucose, plasma electrolytes, $\alpha_{1}$-PI level and complete urine analysis). A signed informed consent form was obtained from all patients prior to entering the study. Patients enrolled in the programme were placed on a standardized bronchodilating therapy, including oral theophylline $300 \mathrm{mg}$ every $12 \mathrm{~h}$, and inhaled salbutamol, $200 \mu \mathrm{g}$ every $8 \mathrm{~h}$, for a 7 day run-in period.

Seven days after the enrolment, patients underwent a second examination to verify the adherence to standardized therapy. Pretreatment urine samples for DES analysis (see below) were collected 2 and 1 day(s) before, and on the morning of the examination. A plasma sample was also collected for pretreatment EDP evaluation (see below). Patients were randomized according to a randomization list, balanced in groups of 10. Drug-treated patients received tablets containing MR889 $500 \mathrm{mg}$ b.i.d.; and the control, placebo-treated group, received lactosefilled tablets b.i.d.

A third examination was conducted 4 weeks after the start of the trial to check the patients' compliance, adherence to protocol and any adverse events. Urine samples were collected 2 and 1 day(s) before and on the morning of the third examination for post-treatment DES measurements and a plasma sample was collected for post-treatment EDP evaluation. All enrolment assessments were repeated.

\section{Urinary desmosine (DES) measurement}

Two millilitre samples of the first morning urine were collected and stored in tightly-sealed screw-cap plastic vials at $-20^{\circ} \mathrm{C}$. The DES analysis was performed in triplicate on $50 \mu \mathrm{L}$ of urine using a radioimmunoassay (RIA) as described previously [17-19]. Urine creatinine was determined by standard automated methodology.

\section{Plasma elastin-derived peptide (EDP) measurement}

Blood was drawn into a vacutainer containing ethylenediamine tetra-acetic acid (EDTA) $0.25 \mathrm{M}$ and $\varepsilon$-amino caproate $1 \mathrm{M}$ dissolved in saline $\mathrm{pH}$ 7.0. The vacutainer was centrifuged and $4 \mathrm{~mL}$ of cell-free plasma collected and transferred to a plastic tube containing N-methylmaleimide $0.35 \mathrm{M}$ and phenylmethyl-sulphonyl-fluoride (PMSF) 0.25 M dissolved in diemethyl sulphoxide (DMSO). The inhibited plasma samples were then frozen at $-70^{\circ} \mathrm{C}$ until needed. The plasma EDP were measured by an enzyme-linked immunosorbent assay (ELISA) as described previously [20].

\section{Statistical analysis}

Data are presented as mean \pm SD. Statistical analysis was performed with analysis of variance (ANOVA) to compare pre- and post-treatment values within drug groups. For between-group comparisons, a multivariate analysis of covariance (MANOVA) was used to adjust for differences in baseline variables. At a p-level equal to or less than 0.05 the difference was taken as being significant. 
Table 1. - Elastin breakdown studies: values of urinary desmosine (DES) and plasma elastinderived peptides (EDP) at entry and at the end of the study.

\begin{tabular}{|c|c|c|c|c|c|c|c|}
\hline \multicolumn{4}{|c|}{ Controls $(\mathrm{n}=28)$} & \multicolumn{4}{|c|}{ Treated $(n=30)$} \\
\hline \multicolumn{2}{|c|}{ 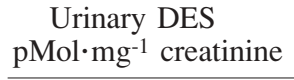 } & \multicolumn{2}{|c|}{$\begin{array}{c}\text { Plasma EDP } \\
\mathrm{ng} \cdot \mathrm{mL}^{-1}\end{array}$} & \multicolumn{2}{|c|}{$\begin{array}{c}\text { Urinary DES } \\
\mathrm{pMol} \cdot \mathrm{mg}^{-1} \text { creatinine }\end{array}$} & \multicolumn{2}{|c|}{$\begin{array}{c}\text { Plasma EDP } \\
\mathrm{ng} \cdot \mathrm{mL}^{-1}\end{array}$} \\
\hline Entry & Final & Entry & Final & Entry & Final & Entry & Final \\
\hline $\begin{array}{r}64.3 \\
\pm 31.6\end{array}$ & $\begin{array}{r}65.6 \\
\pm 34.3\end{array}$ & $\begin{array}{l}38.8 \\
\pm 8.9\end{array}$ & $\begin{array}{r}38.5 \\
\pm 8.8\end{array}$ & $\begin{array}{r}70 \\
\pm 29\end{array}$ & $\begin{array}{r}66.6 \\
\pm 30.6\end{array}$ & $\begin{array}{l}35.1 \\
\pm 8.1\end{array}$ & $\begin{array}{l}36 \\
\pm 9.6\end{array}$ \\
\hline
\end{tabular}

Values are presented as mean \pm SD. All comparisons NS.

\section{Results}

There were no significant differences between the control and treated populations that entered the study. The control group consisted of 27 males and 3 females, mean \pm SD age $65 \pm 7$ yrs (range $45-75 \mathrm{yrs}$ ); and the group of treated patients consisted of 28 males and 2 females, mean \pm sD age $65 \pm 7$ yrs (range $45-75$ yrs). There were no differences between the two groups as far as mean duration of disease ( $13 \pm 6$ yrs for control group and $13 \pm 7$ for treated group ), FEV1 values $(1.30 \pm 0.41 \mathrm{~L}$ for control group and $1.31 \pm 0.48 \mathrm{~L}$ for treated group) and vital capacity (VC) values $(2.62 \pm 0.66 \mathrm{~L}$ for control group and $2.74 \pm 0.73 \mathrm{~L}$ for treated group) at entry were concerned. Also, the two groups did not differ as far as smoking history was concerned $(36 \pm 9$ yrs for control group and $36 \pm 9$ for treated group).

Two patients from the control group withdrew from the study because of mild side-effects, whereas no patient from the MR889-treated group experienced any adverse events.

The value of all the safety parameters were comparatively evaluated before and after the treatments. No statistically significant differences were found in vital parameters (i.e. respiratory and heart rates and blood pressure), blood and urine analysis, electrocardiogram, and pulmonary function tests.

Changes in the level of urinary DES and plasma EDP after treatment were used as putative efficacy parameters. It was found that levels of urinary DES were unchanged at the end of treatment both in controls and in the MR889treated group $(\mathrm{p}=0.64)$. The same results were obtained when plasma EDP levels were considered before and after the treatments $(\mathrm{p}=0.83)$ (table 1$)$.
Although there were no changes in urinary DES and plasma EDP before and after treatments, inspection of individual variations suggested that in some subjects marked changes did occur, especially in urinary DES concentrations. The data were further analysed as a function of the disease heterogeneities. No statistically significant differences were found when patients were analysed either by age or by initial value of FEV1 or smoking history (data not shown ). Patients were also analysed according to the duration of their disease and divided into two subsets, i.e. patients with a disease duration shorter or longer than the mean value (13.7 yrs for controls and 13.3 yrs for the MR889 treated group). The four subsets were homogeneous, with no significant differences with reference to the mean age and initial FEV1 values, but the latter were slightly higher in the subsets of patients with shorter disease duration (table 2). There was no significant difference in the four groups as far as initial and final values of plasma EDP were concerned. In contrast, the values of urine DES dropped significantly at the end of the treatment in the subset of COPD individuals treated with MR889 with a shorter disease duration $(p=0.004)$. Such a change was not seen in the subset of MR889-treated subjects with a longer disease duration, nor in any of the subsets of placebo-treated COPD subjects (table 2).

\section{Discussion}

In this study, we have attempted to evaluate the safety and efficacy of a bench proven effective NE inhibitor in a clinical trial in COPD individuals. To our knowledge, this is the first attempt ever made using a NE synthetic

Table 2. - Characteristics and elastin breakdown markers in the two subsets, $<$ and $\geq$ mean duration of the disease (13.7 yrs for control and $13.3 \mathrm{yrs}$ for MR889-treated subjects).

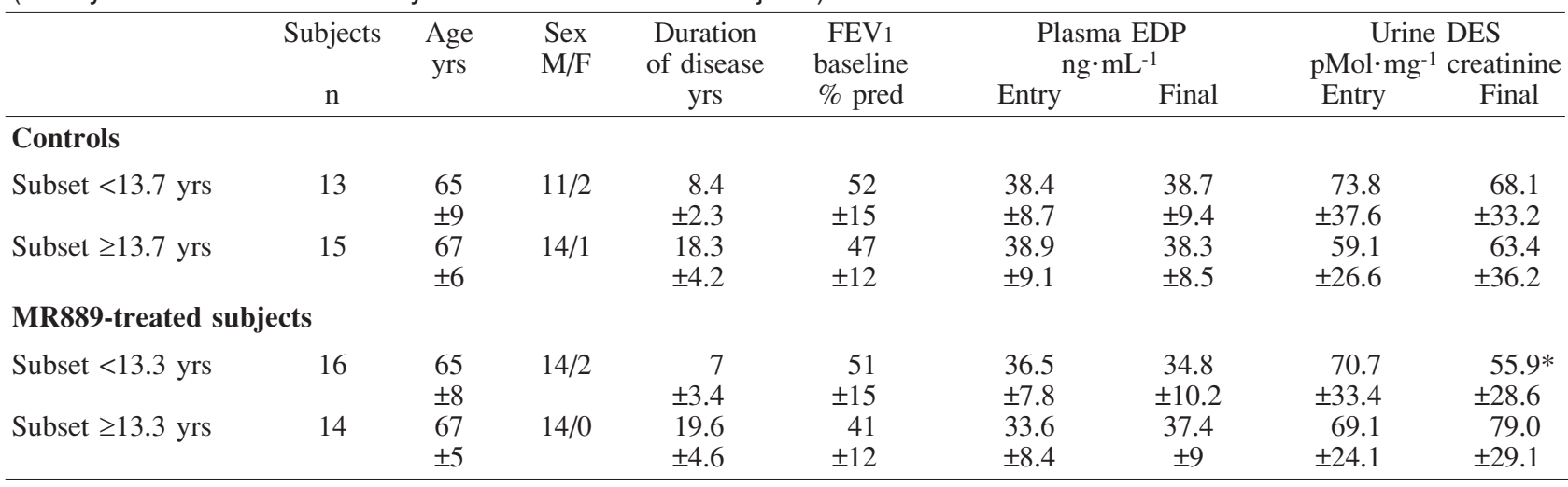

Values are presented as mean \pm SD. M: male; F: female; FEV1: forced expiratory volume in one second; \% pred: percentage of predicted value; EDP: elastin-derived peptides; DES: desmosine. *: $\mathrm{p}=0.004$, compared to level at entry. 
inhibitor. The most definitive means of evaluating the effectiveness of an agent felt to retard or prevent the progression of COPD would be an "outcome study", i.e. a study looking at the reduction of morbidity or mortality. The prohibitive cost, the number of patients involved and the length of such a trial makes this kind of study with a NE inhibitor unreasonable until there is some evidence of its efficacy and safety in humans [21-23]. Such evidence may be gained by looking at changes in the process imputed to cause COPD: the load of NE in the lungs, and the markers of direct elastin destruction [15, 21]. Two of the markers thought to best reflect elastin breakdown are the urine level of elastin-specific desmosine cross-links and plasma EDP fragments [24].

To test the safety and the possible beneficial effects of the orally available NE inhibitor MR889, we chose to design a short-term study in COPD patients, with a pretreatment/post-treatment evaluation of changes in markers of elastin degradation. The investigation was restricted to ex-smoking COPD individuals, since previous experience with colchicine, an agent able to prevent neutrophil degranulation, was effective in reducing the lung NE load in former smokers with COPD, but not in current smokers $[19,25]$.

MR889 may be safely given at an oral dose of 500 $\mathrm{mg}$ b.i.d. for a period of at least 4 weeks to COPD individuals. The drug was well-tolerated, with no major sideeffects or adverse events, and no changes in any of the blood and urine laboratory parameters. Such evidence is consistent with the theory that NE inhibitors belonging to the class of reversible, slow-binding inhibitors, would be well-tolerated $[8,9]$. Safety in long-term MR889 administration remains to be established. However, preliminary data obtained from a study of 600 individuals treated for 8 months with oral MR889 (Media Research Ltd; data on file) seem to confirm the good tolerability and the lack of major adverse events.

We were unable to demonstrate that orally administered MR889 reduced the level of plasma EDP or urine excretion of DES in the total series of COPD patients. This suggests that either MR889 does not modify the breakdown of lung elastin in unselected patients with COPD, or perhaps the negative results were linked to the moderate and slow inhibitory activity of MR889. In fact the $\mathrm{K}_{\text {obsd }} /[\mathrm{I}]\left(\mathrm{M}^{-1} \cdot \mathrm{s}^{-1}\right)$ of MR889 for human NE is 400 (J. Travis, J. Powers, unpublished results), that is about three orders of magnitude lower, for instance, than the value described for the derivative of 7-amino-4-chloro3 -propoxyisocoumarin carrying a $\left(\mathrm{CH}_{3}\right)_{2} \mathrm{CHCH}_{2} \mathrm{CO}$ as 7-substituent [11]. However, to our knowledge, neither isocoumarins nor other mechanism-based or transition state NE synthetic inhibitors have ever been administered to humans.

The data do suggest that in a subset of COPD patients with a relatively short duration of disease, MR889 was able to reduce urine DES excretion. A positive result in the same subset of treated patients was not achieved with plasma EDP. There are no published data concerning a possible correlation between the two markers of elastin breakdown; however, results obtained in two previous trials with colchicine $[19,25]$ seem to support the concept that urine DES may be more sensitive marker of lung destruction than plasma EDP. Urine DES values have been shown to be elevated in cystic fibrosis patients [26], and to drop dramatically after $\alpha_{1}$-PI replacement therapy in a limited number of PIZ individuals [27]. Because of the daily fluctuations of urine DES level in some individuals, the importance of assaying consecutive days of urine has been emphasized [26].

The series of COPD patients with shorter disease duration did not differ from the subset of COPD patients with longer duration of disease, except for a slightly, but not significantly, better level of initial lung function. We can only speculate on reasons why these patients appeared responsive to MR889 treatment. If confirmed in future studies, it may open an important debate about the role of NE in lung destruction in different stages of COPD, and, more importantly, its possible modulation in early stages, even by an inhibitor with moderate activity, such as MR889. Incidentally, our evidence also reinforces the long-standing concept that early therapeutic intervention in COPD is strongly advisable.

In conclusion, this study shows that MR889, a cyclic thiolic neutrophil elastase synthetic inhibitor, can be safely administered to humans. Although we were unable to show a significant reduction in biochemical markers of lung destruction after 4 weeks in unselected patients with chronic obstructive pulmonary disease, there was a significant post-treatment reduction in urine levels of desmosine when the chronic obstructive pulmonary disease patients with relatively short duration of their disease were treated with MR889. Further studies in chronic obstructive pulmonary disease patients stratified by duration of the disease should give a definitive answer as to whether MR889 will reduce the biochemical indices of lung destruction in vivo and whether MR889 efficacy should be tested in a study of outcome.

Acknowledgements: The authors dedicate this paper to the memory of A.B. Cohen and G Quadro. Their continuing support and enthusiasm made this project feasible.

\section{References}

1. Potempa J, Korzus E, Travis J. The serpin superfamily of proteinase inhibitors: structure, function, and regulation. J Biol Chem 1994; 269: 15957-15960.

2. Janoff A. Elastases and emphysema: current assessment of the protease-antiprotease hypothesis. Am Rev Respir Dis 1985; 132: 417-433.

3. Beatty K, Bieth J, Travis J. Kinetics of association of serine proteinase with native and oxidized alpha $a_{1}$-proteinase inhibitor and alpha ${ }_{1}$-antichymotrypsin. J Biol Chem 1980; 255: 3931-3934.

4. Evans MD, Pryor WA. Cigarette smoking, emphysema, and damage to $\alpha_{1}$-proteinase inhibitor. Am J Physiol 1994: 266 (Lung Cell Mol Physiol 10): L593-L611.

5. Stockley RA, Burnett D. Bronchoalveolar lavage and the study of proteinases and antiproteinases in the pathogenesis of chronic obstructive lung disease. Monaldi Arch Chest Dis 1993; 48: 245-253.

6. Snider GL, Stone PJ, Lucey EC. The specific treatment of emphysema. Eur Respir J 1990; 3 (Suppl. 9): 23s-28s.

7. Eriksson S. The potential role of elastase inhibitors in emhysema treatment. Eur Respir J 1991; 4: 1041-1043.

8. Powers JC, Bengali ZH. Elastase inhibitors for treatment of emphysema: approaches to synthesis and biological evaluation. Am Rev Respir Dis 1986; 134: 1097-1100.

9. Baici A, Pelloso R, Horler D. The kinetic mechanism of 
inhibition of human leukocyte elastase by MR889, a new cyclic thiolic compound. Biochem Pharmacol 1990; 39: 919-924.

10. Luisetti M, Piccioni PD, Donnini M, Peona V, Pozzi E, Grassi C. Studies of MR889, a new synthetic proteinase inhibitor. Biochem Biophys Res Commun 1989; 165: 568-573.

11. Powers JC, Kam CM, Hori H, Oleksyszyn J, Meyer EF Jr. Synthetic mechanism-based and transition-state inhibitors for human neutrophil elastase. In: Grassi C, Travis J, Casali L, Luisetti M, eds. Biochemistry of Pulmonary Emphysema. London, Springer-Verlag, 1992: 123-141.

12. Rizzi M, Casale E, Ascenzi P, et al. Crystal structure and NMR investigation of the serine proteinase inhibitor MR889, a cyclic thiolic compound. J Chem Soc Perkin Trans 2 1993: 11; 2253-2256.

13. Rizzi M, Casale E, Coda A, et al. Serine proteinase inhibition by the cyclic thiolic compound YS3025: a crystallographic study. Biochem Int 1992; 28: 385-392.

14. Djinovic Carugo K, Rizzi M, Fasano M, et al. Inhibition of serine proteinases belonging to the chymotrypsin superfamily by the cyclic thiolic compound YS3025: a comparative crystallographic study. Biochem Biophys Res Commun 1993; 193: 32-39.

15. Anthonisen N. Design of a clinical trial to test a treatment of the underlying cause of emphysema. Ann NY Acad Sci 1991; 624S: 31-34.

16. American Thoracic Society. Standards for diagnosis and care of patients with chronic obstructive pulmonary disease (COPD) and asthma. Am Rev Respir Dis 1987; 136: 225-244.

17. King GS, Mohan VS, Starcher BC. Radioimmunoassay for desmosine. Connect Tissue Res 1980; 7: 263-267.

18. Starcher B, Conrad M. A role for neutrophil elastase in the progression of solar elastosis. Connect Tissue Res 1995; 31: 133-140.

19. Cohen AB, Girard W, McLarty J, et al. A controlled trial of colchicine to reduce the elastase load in the lungs of cigarette smokers with chronic obstructive pulmonary disease. Am Rev Respir Dis 1990; 142: 63-72.

20. Kucich U, Christner P, Lippmann M, et al. Utilization of a peroxidase antiperoxidase complex in an enzymelinked immunosorbent assay of elastin-derived peptides in human plasma. Am Rev Respir Dis 1985; 131: 709-713.

21. Snider GL, Stone PJ, Lucey EC. Development and evaluation of antiproteases as drugs for preventing emphysema. In: Grassi C, Travis J, Casali L, Luisetti M, eds. Biochemistry of Pulmonary Emphysema. London, SpringerVerlag, 1992: pp. 144-158.

22. Glass M. The feasibility of an outcome trial in the preventive therapy of emphysema. Ann NY Acad Sci 1991; 624: 195-208.

23. Cohen AB. Clinical trials to treat the underlying cause of emphsyema. Monaldi Arch Chest Dis 1994; 49: 95-97.

24. Rosenbloom J. Biochemical/immunological markers of emphysema. Ann NYAcad Sci 1991; 624s: 7-12.

25. Cohen AB, Girard W, Mclarty J, et al. A controlled trial of colchicine to reduce the elastase load in the lungs of ex-cigarette smokers with chronic obstructive pulmonary disease. Am Rev Respir Dis 1991; 143: 1038-1043.

26. Starcher B, Green M, Scott M. Urinary desmosine as an indicator of acute pulmonary disease. Respiration 1995; 62: 252-257.

27. Stone PJ, Morris III TA, Franzblau C, Snider GL. Preliminary evidence that augmentation therapy diminishes degradation of cross-linked elastin in alpha- ${ }_{1}$-antitrypsin deficient humans. Respiration 1995; 62: 76-79. 